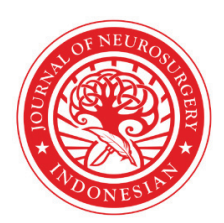

\title{
The incidence of surgical site infections in neurosurgery procedure during January - December of 2017 in Kariadi General Hospital, indonesia
}

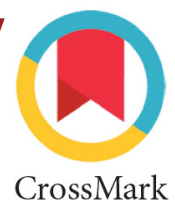

\author{
Deviana Restu Rerung ${ }^{1 *}$, Erie B.P Setya Budi Andar ${ }^{2}$
}

${ }^{1}$ General Surgery Resident, Diponegoro University / Kariadi General Hospital, Semarang, Central Java, Indonesia ${ }^{2}$ Staff of Neurosurgery Department, Diponegoro University / Kariadi General Hospital, Semarang, Central Java, Indonesia
*Corresponding to: Deviana Restu Rerung; General Surgery Resident, Diponegoro University / Kariadi General Hospital, Semarang, Central Java, Indonesia; dev_20_87@yahoo.com

Received: 2019-12-14 Accepted: 2020-05-09 Published: 2020-11-19

\section{ABSTRACT}

Introduction: Surgical site infection (SSI) remains as substantial cause of morbidity, prolonged hospitalization and death. The most common types of inpatient surgical procedures include neurosurgery procedures. The aim of this study is to provide the incidence of surgical site infection in neurosurgical procedure and determine the most common etiological agent of surgical site infection in Kariadi General Hospital period January till December of 2017.

Methods: This was a descriptive-retrospective study. Data were collected from medical record of patient who underwent neurosurgical intervention in Kariadi General Hospital between 1 January - 31 December 2017. All respondent with neurosurgery procedure that meets the criteria of National Nosocomial Infections Surveillance (NNIS) of superficial incisions and Hulton criteria were included.

Results: A total of 196 patients underwent neurosurgery procedure during between 1 January - 31 December 2017. Of these, 15 cases $(7.65 \%)$ experienced surgical site infections. There were 6 female cases and 9 male cases (2:3). The highest incidence rate was in old age group(45-65 yo). In microbiology study, we found some bacteria such as Acinetobacter baumannii 2 cases (13.3\%), Escherichia coli 4 cases (26,6\%), Enterococcus faecalis 1 case $(6,6 \%)$, Proteus mirabilis 1 case (6,6\%), no-bacteria 6 cases (40\%) and 1 unidentified (6,6\%).

Discussions: Surgical site infections are frequent and serious complications of surgical procedures and remain a major issue for patient safety. Several independent predictors of SSI is commonly in older people, including comorbid conditions, perioperative variables and socioeconomic factors. In an analysis of etiologic agents of infections in this study is Escherichia coli. According to the literature that the responsible pathogens in most SSIs are originate from the patient's endogenous flora, in addition of exogenous resources. Its important to follow the evidence-based guidelines for the prevention of SSIs, from preoperative, perioperative and postoperative procedure.

Conclusion: We found 15 cases of surgical site infection in neurosurgery procedure in 1year period of time. Numerous patientrelated and procedure-related factors influence the risk, and it requires a bundle approach to prevent the SSI incidence

Keywords: surgical site infection, neurosurgery, infection

Cite This Article: Rerung, D.R., Andar, E.B.P.S.B. 2020. The incidence of surgical site infections in neurosurgery procedure during January December of 2017 in Kariadi General Hospital, indonesia. Indonesian Journal of Neurosurgery 3(3): 88-91. D0I: 10.15562/ijn.v3i3.98

\section{INTRODUCTION}

Surgical site infections (SSIs) are prevalent around the world, which are a serious and undesirable outcome of surgery. SSI is the most frequently reported health care-associated infections (HAIs) from health care facilities in low- and middleincome countries. ${ }^{1}$ The CDC health care-associated infections (HAIs) prevalence survey found that there were an estimated 110.800 surgical site infection (SSI) associated with impatient surgeries in 2015. Surgical site infections remain a substantial cause of morbidity, prolonged hospitalization and death. SSI is associated with mortality rate 3\% and $75 \%$ of SSI associated death are directly attributable to the SSI. ${ }^{2}$

Surgical site infection (SSI) defined by the Centers for Disease Control and Prevention (CDC) as infection related to an operative procedure that occurs at or near surgical incision within 30 days of the procedure or within 90 days if prosthetic material is implanted at surgery, is among the most common preventable complication after surgery. ${ }^{1}$ Superficial infections may cause minimal disruption in recovery, while deep tissue infection can hinder healing, can prolong length of stay in hospital and may result in loss of mobility, lack of recovery to presurgical status and even increase death rate. The most common types of inpatient surgical procedures include cesarean section, orthopedic procedures (hip and knee replacement, hip fracture repair), neurosurgical procedures (spinal fusion and laminectomy) and intraabdominal procedure (cholecystectomy and colorectal resections)., ${ }^{3,4}$

There were lack of data of surgical site infection in neurosurgical procedure in Kariadi Hospital. The aim of this study is to provide the incidence of 
surgical site infection in neurosurgical procedure and determine the most common etiological agent of surgical site infection in Kariadi General Hospital period January till December of 2017.

\section{METHODS}

This was a descriptive-retrospective study. Data were collected from medical record of patient who underwent neurosurgical intervention in Kariadi General Hospital between 1 January - 31 December 2017.

All respondent with neurosurgery procedure that meets the criteria of National Nosocomial Infections Surveillance (NNIS) of superficial incisions and Hulton criteria were included. Additional criteria included clinical signs of infection plus evidence of infection at the surgical site (pus, wound dehiscence, erythema or cellulitis).

Cases were excluded from the study if they were admitted with primary cranial or spinal infections (eg abscess, apmyeme) or patients with multiple operations performed at the same site for reasons other than SSI were also excluded to avoid duplicates in the data.

\section{RESULTS}

A total of 196 patients underwent neurosurgery procedure during between 1 January - 31 December 2017. Of these, 15 cases (7.65\%) had surgery for suspected infection and meets the inclusions criteria. 181 cases were excluded from this study.

There were 6 female cases and 9 male cases (2:3). The samples were divided into age groups, child 3 cases (20\%), adolescent 4 cases (26,6\%), adult 2 cases $(13.3 \%)$ and old age 6 cases (40\%). The highest incidence rate was in old age group.

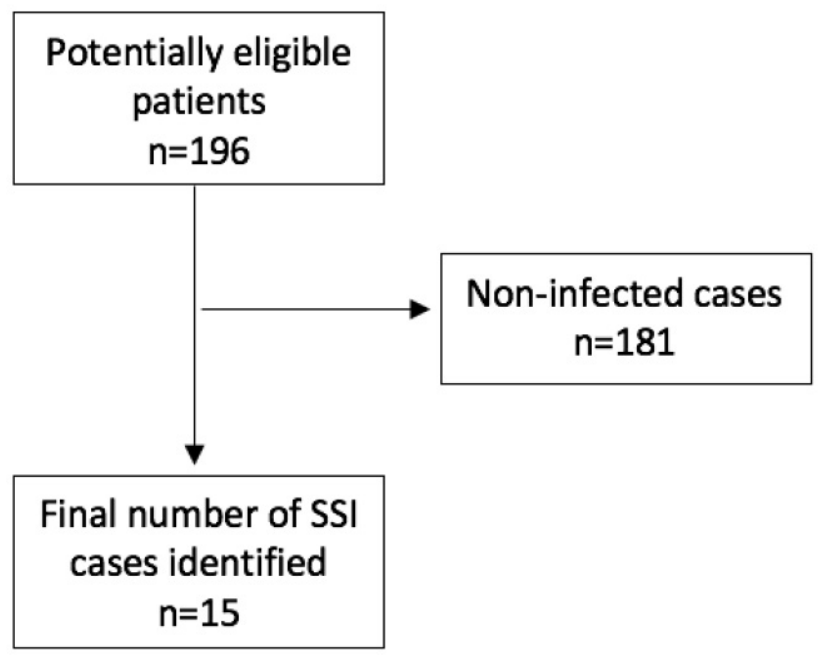

Figure 1. Summary of patient selection
In microbiology study, we found some bacteria such as Acinetobacter baumannii 2 cases (13.3\%), Escherichia coli 4 cases (26,6\%), Enterococcus faecalis 1 case $(6,6 \%)$, Proteus mirabilis 1 case $(6,6 \%)$, nobacteria 6 cases (40\%) and 1 unidentified (6,6\%). We found Escherichia coli as the most common cause of surgical site infection in neurosurgery procedure in 2017. 1 case did not underwent the microbiology culture.

\section{DISCUSSION}

Surgical site infections are frequent and serious complications of surgical procedures and remain a major issue for patient safety despite improvements in surgical practice and infection control techniques. They are associated with a prolonged duration of hospitalization, readmissions, and reinterventions and the death. ${ }^{5}$

Among 15 cases of surgical site infection in neurosurgery procedure in Kariadi General Hospital during January-December of 2019, the highest incidence rate was in old age group old age, 6 cases (40\%) and most common in male. It is all more significant that in the patient population observed, the characteristics of patients changed significantly, especially regarding their age, which may indicate a trend of an aging patient population and their increased susceptibility to infections. At the same time, the next property that the researcher has no control over is sex, which among the patients operated on, men were predominant, and they also usually more vulnerable to infections after neurosurgical procedures. ${ }^{6,7}$ The fact that being a man greatly affects the risk of SSI was also reported in Poland thoracic procedures. ${ }^{8}$

SSI commonly complicates surgical procedures in older people and is associated with substantial attributable mortality and costs. ${ }^{9}$ Because the population is progressively aging, the proportion of the population older than 65 years is projected to increase $12,4 \%$ to $19.6 \%$ by $2030 .{ }^{10}$ Previous studies have identified several independent predictors of SSI in older people, including comorbid conditions, perioperative variables and socioeconomic factors. ${ }^{9}$

In an analysis of etiologic agents of infections in this study is Escherichia coli. According to the literature that the responsible pathogens in most SSIs are originate from the patient's endogenous flora. The most commonly isolated organisms are S. aureus, coagulase-negative staphylococci, Enterococcus spp. and Escherichia coli, however the pathogens isolated depend on the procedure. Pathogens may also originate from the operative site, particularly in patients undergoing insertion of a prosthesis or other implant. ${ }^{10}$

In addition to the patient's endogenous flora, 
Table 1. Summarize of patient demographic

\begin{tabular}{llcc}
\hline \multicolumn{1}{c}{ Study subject } & \multicolumn{1}{c}{ Characteristics } & n & Percentage \\
\hline Sex & Male & 9 & $60 \%$ \\
Age & Female & 6 & $40 \%$ \\
& Child (0-16 yo) & 3 & $20 \%$ \\
& Adolescent (17-25 yo) & 4 & $26,6 \%$ \\
& Adult (26-45 yo) & 2 & $13,3 \%$ \\
Pre-operative & Old age (45-65 yo) & 6 & $40 \%$ \\
diagnose & Fracture & 4 & $26.7 \%$ \\
Microbiology culture & Non-fracture & 11 & $73.3 \%$ \\
& Acinetobacter baumannii & 2 & $13,3 \%$ \\
& Escherichia coli & 4 & $26,6 \%$ \\
& Enterococcus faecalis & 1 & $6,6 \%$ \\
& Proteus mirabilis & 1 & $6,6 \%$ \\
& No-bacterial & 6 & $40 \%$ \\
& Unidentified & 1 & $6,6 \%$ \\
\hline
\end{tabular}

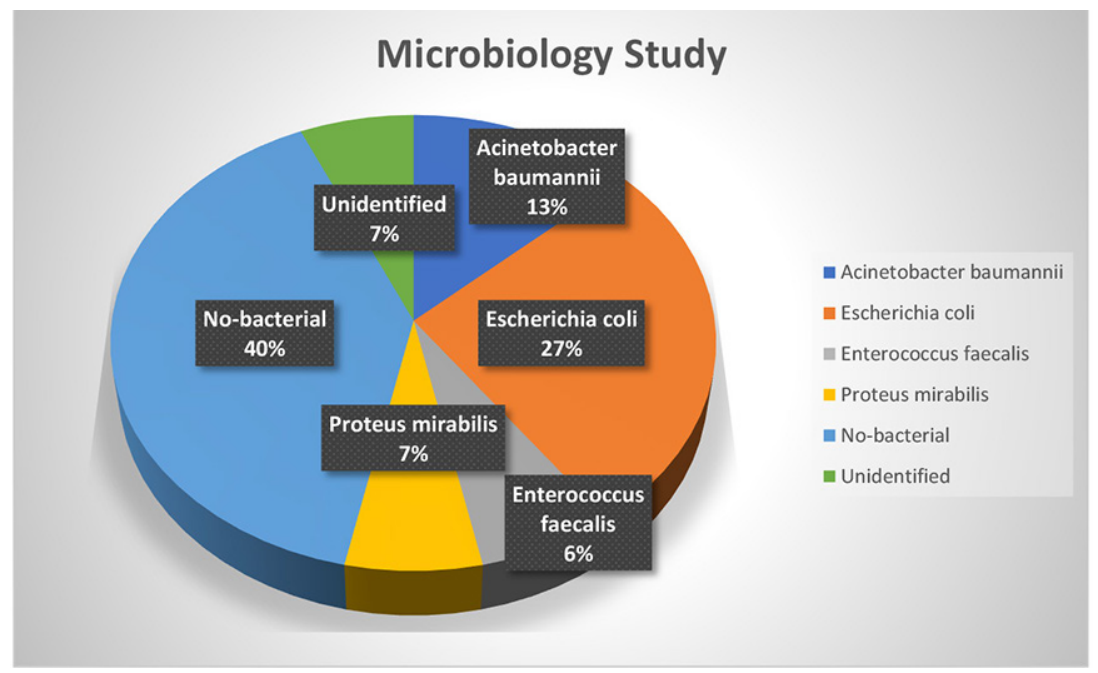

Figure 2. The result of microbiology study

SSI pathogens may originate from exogenous sources such as members of the surgical team, the operating theatre environment and instruments and materials brought within the sterile field during the procedure. Such pathogens are predominantly aerobes, particularly Gram-positive organisms such as staphylococci and streptococci. ${ }^{11}$

Understanding the burden of SSIs as well as significant risk factor can help us improve our practice with respect to preventing them. This study strengthens the knowledge that various risk factors are associated with the development of SSIs, with few of these factors being modifiable. Smoking, diabetes, operative duration, all have been shown to inhibit satisfactory wound healing which could in turn lead to wound heals. In addition, poor surgical technique may also be considered a risk factor as a more watertight closure may help prevent wound leaks. ${ }^{12,13}$
Numerous patient-related and procedurerelated factors influence the risk of SSI, and hence prevention requires a bundle approach, with systemic attention to multiple risk factors, in order to reduce the risk of bacterial contamination and improve the patient's defences. Evidence-based guidelines for the prevention of SSIs have been published by the CDC. Preoperative strategies focus on controlling patient related risk factors and appropriate hand/ forearm antisepsis for surgical team members. Preexisting infections at sites remote from the operation site should be identified and treated. Short courses of antimicrobial prophylaxis are widely used to reduce SSI risk. The choice of agent should be based on the pathogens most commonly associated with the procedure being performed. Also surgical personnel should undertake a thorough surgical scrub before donning surgical gowns and gloves. ${ }^{11}$

The CDC guidelines emphasis the importance of surgical technique and aseptic precautions for the preventions of SSIs. Good surgical technique requires attention to the maintenance of haemotasis, removal of devitalized tissue and foreign bodies as completely as possible, and elimination of dead space at the surgical site. Gloves, facemasks, caps, gowns and sterile drapes should be used to minimize transmission of potential pathogens to the wound. It should be noted that despite precautions such as these, some contamination of the surgical site is inevitable because some endogenous bacteria remain even after excellent preoperative preparation of the site. ${ }^{9,14}$

The risk of SSI can persist for up to 30 days after a surgical operation or for as long as one year after an operation in which the patient is given an implant. CDC guideline recommend that incisions that have been closed by primary intention should be protected by sterile dressings for $24-48$ hours, and that personnel should use sterile technique when changing dressings on any kind of skin incision. ${ }^{9}$

A possible limit of our study could be the small sample size. Those results strengthen the need for interventional studies to reduce the risk of SSIs in neurosurgical procedure in order to produce cost savings, reduce mortality risk and improve functional status. ${ }^{9}$

\section{CONCLUSION}

Our retrospective study showed among 196 patients underwent neurosurgery procedure in 2017, 15 cases $(7.65 \%)$ experienced surgical site infection. Most common affected in male and old age group. The most common etiologic agent was Eschericia coli. 
It is important to recognize that morbidity and mortality associated with SSIs is preventable. Numerous patient-related and procedure-related factors influence the risk of SSI, and hence prevention requires a bundle approach, with systemic attention to multiple risk factors, in order to reduce the risk of bacterial contamination and improve the patient's defences.

\section{CONFLICTS OF INTEREST}

The author declare no clonflict of interest.

\section{FUNDING}

This article received no specific funding from any funding agency in the public, commercial, or notfor-profit sectors.

\section{AUTHORS' CONTRIBUTIONS}

Authors took part in design of the study, contributed to data collection, participated in writing the manuscript and all agree to accept equal responsibility for accuracy of the contents of this article

\section{REFERENCES}

1. Centers for Disease Control and Prevention, National Healthcare Safety Network. CDC/NHSN Surveillance Definitions for Specific Types of Infections. Jan 2017. Accessed November 21, 2019 : https://www.cdc.gov/nhsn/ pdfs/pscmanual/17pscnosinfdef_current.pdf.

2. Magill, S.S., et al., "Changes in Prevalence of Health CareAssociated Infection in U.S. Hospitals". New England Journal of Medicine, 379(18): (2018): 1732-44.

3. Kristen A. Ban, M, Joseph P. Minei, Christine Laronga, American College of Surgeons and Surgical Infection Society: Surgical Site Infection Guidelines, 2016 Update Presented at the Surgical Infection Society, Florida, January 2017 Volume 224, Issue 1, Pages 59-74 Available from: https://www.journalacs.org

4. Mangram A J, Horan T C, Pearson M L,Silver L C, Jarvis W R.1999. Guidline for prevention of Surgical Site of Infection. Columbia University School of Nursing;New York
5. Crolla RMPH, van der Laan L, Veen EJ, Hendriks Y, van Schendel C, Kluytmans J. Reduction of surgical site infections after implementation of a bundle of care. PLoS One. 2012;7(9): e44599

6. McCutcheon, B.A.; Ubl, D.S.; Babu, M.; Maloney, P.; Murphy, M.; Kerezoudis, P.; Bydon, M.; Habermann, E.B.; Parney, I. Predictors of Surgical Site Infection Following Craniotomy for Intracranial Neoplasms: An Analysis of Prospectively Collected Data in the American College of Surgeons National Surgical Quality Improvement Program Database. World Neurosurg. 2016, 88, 350-358. [CrossRef] [PubMed]

7. Fang, C.; Zhu, T.; Zhang, P.; Xia, L.; Sun, C. Risk factors of neurosurgical site infection after craniotomy: A systematic review and meta-analysis. Am. J. Infect. Control 2017, 45, e123-e134. [CrossRef]

8. Dubiel, G.; Rogozin 'ski, P.; Z $\square$ aloudik, E.; Brulin'ski, K.; Róz $\square$ an 'ska, A.; Wójkowska-Mach, J. Identifying the Infection Control Areas Requiring Modifications in Thoracic Surgery Units: Results of a Two-Year Surveillance of Surgical Site Infections in Hospitals in Southern Poland. Surg. Infect. 2017, 18, 820-826

9. Kaye KS, Sloane R, Sexton DJ, Schmader KA. Risk factors for surgical site infections in older people. J Am Geriatr Soc 2006; 54(3):391-396

10. US Bureau of the Census. Projected population of the United States, by age and sex: 2000 to 2050. US Census Bureau 2004b. Accessed November 9, 2014. Available from: http://www. census.gov/population/projections/data/ national/ usinterimproj.html

11. ngramAJ,HoranTC,PearsonML,SilverLC, JarvisWR; Hospital Infection Control Practice Advisory Committee. Guideline for prevention of surgical site infection, 1999. Infect Control Hosp Epidemiol 1999;20:247 278.

12. Uzuka T, Takahashi H, Nakasu $Y$ et al. Surgical site infection after malignant brain tumor resection: a multicenter study for induction of a basic care bundle. Neurol Med Chir 2017; 57: 542-547.

13. Karhade AV, Cote DJ, Larsen AM, Smith TR. Neurosurgical infection rates and risk factors: a National Surgical Quality Improvement Program analysis of 132,000 patients, 20062014. World Neurosurg 2017; 97: 205-212.

14. Osler T. Antiseptics in surgery. In: Fry DE, Ed. Surgical infections, 1st edn. New York: Little, Brown, \& Co.; 1995, pp. 119125.

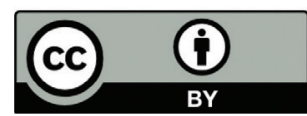

This work is licensed under a Creative Commons Attribution 